\title{
Le rire unit, il est communicatif, il apporte des instants de bonheur...
}

\author{
Ursula Steiner-König \\ Dr med., déléguée de la FMH au Comité central de la Journée des malades
}

L’idée de placer la prochaine Journée des malades sous le signe de l'humour a été accueillie avec enthousiasme par les membres du comité de la Journée des malades: un thème pour une fois positif qui ne se focalise pas sur la maladie! Quelque chose d'accessible à tous! Facile à dire, mais comment faire pour illuminer d'un sourire le visage d'une personne gravement malade? Pas question de faire des plaisanteries, car je ne sais pas si la personne en face de moi partage le même humour. L'idée n'est pas non plus de déclencher un fou rire, et encore moins de verser dans le sarcasme ou le cynisme, au risque de dévaloriser, de blesser la personne ou d'empêcher la rencontre avec l'autre. Ne l'oublions pas: l'humour et le rire sont deux choses différentes! "To analyse humour is a task as delicate as analysing the composition of a perfume with its multiple ingredients, some of which are never consciously perceived while others, when sniffed in isolation, would make one wince.» Voilà ce que l'on peut lire dans les commentaires à la rubrique «humour» de l'Encyclopædia Britannica. Le rire, quant à lui, est une manifestation biologique visant uniquement à relâcher les tensions. Le lien entre les deux pourrait être caractérisé par ce que l'écrivain allemand Wilhelm Raabe aurait dit de l'humour: «il est la bouée sur le fleuve de la vie.»

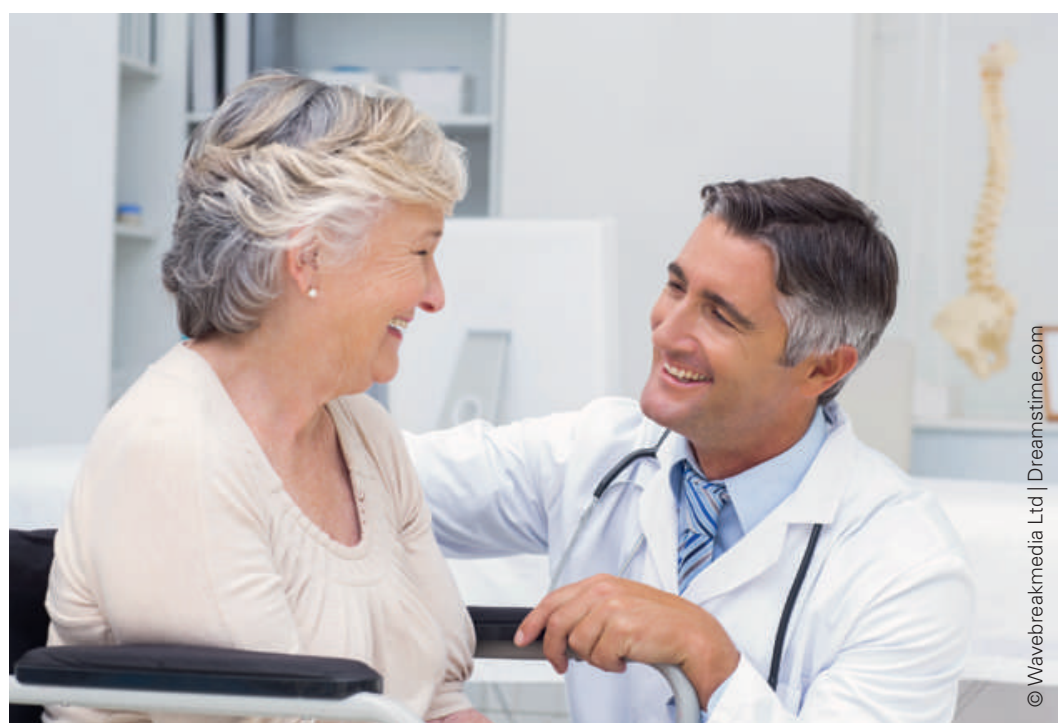

Le rire détend et libère les pensées.
Ce à quoi on aspire, c'est offrir quelques instants de bonheur à un malade en essayant de le soulager et de lui transmettre une lueur d'espoir déclenchant un sourire d'apaisement. Mais comment y parvenir? En lui transmettant avec douceur une perspective nouvelle (voire même inédite) afin de créer des émotions positives et d'alléger ses pensées, à l'instar de la réaction d'un bébé à qui l'on sourit. (Nous savons que le rire est généré dans une région du cerveau qui se développe bien avant le centre de la parole.) Le sourire devient alors un signe d'acceptation, de compréhension, de rencontre authentique.

La recherche sur l'humour relève aussi l'implication de certaines régions du cortex cérébral qui n'atteignent leur maturité que tard dans le développement. Ce qui veut dire que les fonctions corticales supérieures ne jouent pas un rôle anodin (cf. par ex. Barbara Wild).

\section{L’aspect salutogénique}

Nous ne savons pas à partir de quand l'aspect salutogénique du rire a été non seulement identifié, mais également décrit et systématisé dans la pratique. En revanche, il existe depuis une vingtaine d'années une augmentation des publications sur les nombreux bénéfices de l'humour, du sourire, du rire ou du fou rire. Le recours à l'humour est ainsi analysé dans différents contextes, par exemple auprès de patients souffrant de douleurs ou de démences, d'enfants gravement malades, dans les soins palliatifs ou encore le travail social. En réalité, ses effets relaxants, apaisants et déstressants peuvent se déployer partout où il y a interaction entre plusieurs personnes. Comme le dit le satiriste suisse alémanique Andreas Thiel: «l'humour est la forme de conscience la plus élevée», et de préciser «l'humour permet de prendre conscience que rien n'est aussi sérieux qu'il n'y paraît.» En 1998, à l'occasion du $3^{\mathrm{e}}$ congrès bâlois de l'humour, des spécialistes de la psychologie positive ont fondé l'association germanophone HumorCare, divisée en 2001 en différentes sociétés nationales indépendantes. Entretemps, deux autres fondations, Humor \& Gesundheit et Théodora se sont fait connaître en Suisse et mettent l'accent sur des programmes différents. 
Il est par ailleurs intéressant de noter que dès le début des années 1990, un médecin indien, Madan Kartaria, avait pris conscience des bienfaits du rire (à distinguer de l'humour) sur la santé, donnant ainsi naissance à des clubs de rire accessibles à tous qui se tenaient à l'origine dans des lieux publics: des offres de prévention susceptibles en plus de faire économiser des coûts. Selon lui, le mécanisme même du rire, c'est-à-dire le fait de rire de manière intentionnelle, serait plus efficace que de raconter des blagues.

Le sourire devient alors un signe d'acceptation, de compréhension, de rencontre authentique.

Sur le plan physiologique, on sait que le rire stimule non seulement la musculature du visage, mais également le diaphragme et les muscles du ventre. Le rythme cardiaque et l'approvisionnement en oxygène augmentent; les hormones liées au stress diminuent, et le cerveau secrète une quantité accrue d'endorphine (l'hormone du bonheur). Selon une étude, 20 secondes de rire représentent une performance physique équivalente à trois minutes d'aviron rapide (même si ce n'est pas l'effet recherché chez les malades).

Au chevet du patient, il faut faire preuve de beaucoup de tact et d'empathie pour arriver à lui remonter le moral. Les moqueries, les remarques dégradantes ou blessantes n'y ont pas leur place. Dans le cadre de la psychologie de la personnalité, l'Institut de psychologie de l'Université de Zurich étudie depuis 1980 les différences de perception de l'humour en fonction de la personnalité. On a ainsi découvert que les personnalités extraverties riaient davantage et plus facilement. Les personnes de nature joyeuse évoquent quant à elles moins de symptômes physiques et supportent mieux le stress.

\section{Le potentiel psychologique de l'humour}

On distingue (selon Salameh) trois dimensions de l'effet thérapeutique de l'humour:

Emotionnelle: disparition des inhibitions, encouragement de la verbalisation, permet un échange immédiat et spontané des sentiments.

Cognitive: mobilisation de la créativité, sensibilisation aux nouvelles situations, aide à remplacer des comportements rigides par des comportements plus souples. Communicationnelle: effet relaxant, stimulant et bénéfique pour les relations, favorise un climat d'ouverture et d'égalité.

Une étude non encore publiée de l'Institut de psychologie de l'Université de Zurich auprès de personnes atteintes de démence montre par exemple qu'un sourire qui vient du cœur se distingue des formes moins spontanées de rire ou de sourire. (Un rapport de CURAVIVA à ce sujet est disponible sur le site internet de la Journée des malades)

\section{L'humour des médecins}

Si l'humour noir, les plaisanteries crues et/ou sarcastiques échangées entre confrères fonctionnent comme un exutoire et permettent de rendre supportables les situations souvent difficiles rencontrées au quotidien, ce type d'humour serait totalement déplacé au chevet des malades. Comme l'évoquait Samia Hurst dans un numéro du BMS paru à l'automne 2015 en parlant de la "part sombre» de l'humour médical: «nous ne devons surtout pas en avoir honte", mais nous devons avoir conscience de son caractère particulier et connaître ses limites. Une situation que rencontrent certainement aussi les humoristes, pour qui le rire n'est pas innocent. Comme l'admet le satiriste Andreas Thiel, «il est plus facile pour moi d'écrire quelque chose de drôle sur la torture, la guerre ou le terrorisme que sur l'humour.» Nous voilà revenu à notre point de départ!

Aucun guide de bonnes pratiques n'existe pour savoir comment nous comporter et trouver le bon ressenti, le bon parfum (si on se réfère à la définition anglaise de l'humour) face à une personne malade et souffrante. Les facteurs mobilisés sont multiples et relèvent à la fois de la biologie, de la psychologie et de la sociologie. C'est pourquoi la nouvelle approche présentée par B. Kissling dans le Swiss Medical Forum, celle de la «qualité qualitative», mérite d'être saluée. Une collaboration avec les sciences humaines et sociales bénéficierait en effet non seulement aux malades mais également aux soignants, par un soupçon de poésie, une dose d'harmonie et peutêtre aussi un brin de mélodie...

\section{Références}

- Michael Titze Hrsg. Kleinbasel und der «Humor in der Therapie»; HCD-Verlag, Tuttlingen, 2013: plusieurs articles.

- Samia Hurst. Si on ne peut même plus rigoler; BMS 2015;96(40):1466.

- Daniele Muscionico. Interview avec Andreas Thiel. NZZ 6 nov. 2015

http://www.nzz.ch/feuilleton/humor-die-hoechste-form-vonerkenntnis-1.18641881

- Barbara Wild Hrsg. Humor in Psychiatrie und Psychotherapie; Schattauer-Verlag Stuttgart, 2012, notamment la citation de Wilhelm Raabe.

- Salameh W. cité dans Peter Hain, Humor als therapeutische und soziale Kompetenz. www.humor.ch/phain/phkompetenz.htm

- Madan Kataria: www.humor.ch ou www.laughteryoga.com

- Bruno Kissling. Qualité qualitative. Swiss Med Forum. 2015;(50-51):1172-3.

- Leuenberger Beat. Fahnden nach dem Duchenne-Lächeln. Curaviva 7-8/13.

- Site internet de la Journée des malades: www.tagderkranken.ch 